\title{
Blood pressure variability and prognosis in acute ischemic stroke with vascular compression on the rostral ventrolateral medulla (RVLM)
}

\begin{abstract}
Shiro Aoki, Toshiho Ohtsuki, Naohisa Hosomi, Yoshimasa Sueda, Tomoyuki Kono, Takemori Yamawaki and Masayasu Matsumoto

One of the known causes of hypertension is vascular compression on the rostral ventrolateral medulla (RVLM). However, it remains unknown whether RVLM vascular compression causes the significant variability in blood pressure observed during acute ischemic stroke. The purpose of this study was to evaluate differences in blood pressure variability and prognosis in acute ischemic stroke patients based on the presence or absence of RVLM vascular compression. We evaluated $\mathbf{5 6}$ patients with acute ischemic stroke. Blood pressure was measured every $6 \mathrm{~h}$ for $72 \mathrm{~h}$ after admission and evaluated with successive variation (SV). The presence of RVLM vascular compression was evaluated using time-of-flight 3D magnetic resonance imaging. Neurological severity was evaluated using the National Institutes of Health Stroke Scale (NIHSS) at admission and 14 days after admission, and clinical improvement was determined by taking the difference in the NIHSS scores between admission and at 14 days.

Patient clinical outcome was evaluated with the modified Rankin scale on discharge. Vascular compression of the RVLM was identified in 15 patients $(26.8 \%)$. The proportion of patients showing clinical improvement was significantly higher in the noncompression group (odds ratio, $0.21(95 \% \mathrm{Cl}=0.06-0.78) ; P=0.01)$. The $\mathrm{SV}$ value for systolic blood pressure was significantly higher in the compression group $(P<0.0001)$. We found that patients with RVLM vascular compression had a greater variability in blood pressure during the acute ischemic stroke phase, which may be related to poorer prognosis.
\end{abstract}

Hypertension Research (2011) 34, 617-622; doi:10.1038/hr.2011.17; published online 24 February 2011

Keywords: acute ischemic stroke; blood pressure; rostral ventrolateral medulla

\section{INTRODUCTION}

Regardless of nationality or ethnicity, hypertension is a lifestyle-related disease afflicting patients throughout the world and is a major risk factor for stroke. Hypertension is a multifactorial disease. It has been reported that a large population of patients with vascular compression on the rostral ventrolateral medulla (RVLM) have hypertension. ${ }^{1-4}$ Surgical decompression of the RVLM reduced sympathetic nerve activity and normalized systemic blood pressure. ${ }^{5,6}$ Therefore, it is suggested that vascular compression on the RVLM influences the development or maintenance of hypertension. The RVLM has been experimentally shown to be a site of cardiac and vasomotor regulation. The mechanism of increased blood pressure in patients with vascular compression on the RVLM remains to be completely elucidated. It is currently hypothesized that chronic stimulation of this region with vascular compression can cause constitutive activation of the sympathetic nervous system and the development of hypertension. ${ }^{7,8}$

More than $80 \%$ of acute stroke patients have elevated blood pressure. Several days following the incidence of stroke, however, the blood pressure in these patients returns to baseline levels. ${ }^{9}$ This blood pressure elevation varies depending on the subtype of ischemic stroke and on the patient's medical history. ${ }^{10}$ In general, the cause of elevated blood pressure during the acute phase of ischemic stroke is presumed to be an increase in sympathetic nerve activity and stress from the ischemic insult that disrupts intracerebral autoregulation to maintain cerebral blood flow. ${ }^{11}$ During acute ischemic stroke, the rapid decrease in blood pressure reduces cerebral blood flow in parallel to a decrease in the perfusion pressure that is sufficiently large to expand infarct volumes and worsen neurologic symptoms. Therefore, such an excessive lowering of blood pressure during acute ischemic stroke is not desirable. Even in studies examining the correlation between blood pressure and prognosis in the acute ischemic stroke phase, the variability in blood pressure has been shown to be an independent prognostic factor for a poor outcome. ${ }^{12,13}$

RVLM vascular compression may cause significant variability in blood pressure during acute ischemic stroke by sympathetic nerve activation. ${ }^{14}$ However, to our knowledge, it remains unclear whether RVLM vascular compression influences blood pressure during acute ischemic stroke. Therefore, the purpose of this study was to evaluate differences in blood pressure variability and prognosis during acute ischemic stroke in the presence or absence of RVLM vascular 
compression and to examine the influence of chronic sympathetic nerve activation on acute ischemic stroke.

\section{METHODS}

\section{Subjects and study design}

This study was conducted in consecutive patients with acute ischemic stroke admitted between April 2008 and March 2010 to Hiroshima University Hospital within $24 \mathrm{~h}$ of stroke onset. Exclusion criteria included the inability to undergo magnetic resonance imaging (MRI) examination, the administration of thrombolysis, an National Institutes of Health Stroke Scale (NIHSS) score $\geqslant 23$ on admission, the use of anti-hypertensive or vasopressor medicines within $72 \mathrm{~h}$ after admission, and surgery within $72 \mathrm{~h}$ after admission. In addition, we excluded patients with medullary infarctions, which might have effects on RVLM. The stroke subtype was determined based on MRI findings, electrocardiography, and carotid artery and cardiac ultrasound findings by at least two stroke specialists according to the Trial of Org 10172 in Acute Stroke Treatment (TOAST) classification. ${ }^{15}$ The acute treatment was determined based on the stroke subtype in accordance with the established guidelines. Neurological severity was evaluated using the NIHSS upon admission and 14 days after admission. ${ }^{16}$ Their clinical outcome was evaluated with the modified Rankin scale at the time of discharge. ${ }^{17}$ The study was approved by the institutional review board of Hiroshima University Hospital.

Hypertension was defined as the use of any anti-hypertensive medicines before admission or a confirmed blood pressure of $\geqslant 140 / 90 \mathrm{~mm} \mathrm{Hg}$ at rest after 2 weeks from stroke onset. Diabetes mellitus was defined as $\mathrm{HbAlc}$ $\geqslant 6.5 \%$, fasting blood sugar $\geqslant 126 \mathrm{mg} \mathrm{dl}^{-1}$, or the use of any anti-diabetic medicines. Hyperlipidemia was defined as total cholesterol $\geqslant 220 \mathrm{mg} \mathrm{dl}^{-1}$, lowdensity lipoprotein cholesterol $\geqslant 140 \mathrm{mg} \mathrm{dl}^{-1}$ on admission, or the use of any anti-hyperlipidemic medications. For $72 \mathrm{~h}$ after admission, blood pressure and pulse rate were measured every $6 \mathrm{~h}$ on the unaffected arm in a resting supine position using a standard automated sphygmomanometer without any antihypertensive medicines. Blood pressure and pulse rate variability was evaluated with successive variation (SV), defined as the square root of the averaged squared differences between two successive measurements. ${ }^{18}$

The presence or absence of RVLM vascular compression was evaluated on a 3T MRI unit (GE, Fairfield, CT, USA) using 3D time-of-flight (slice thickness of $1.0 \mathrm{~mm}$, TR/TE/flip angle, $23 / 3.4 / 18^{\circ}$ ). The RVLM was located at the rootentry zone of cranial nerves IX and X (Figure 1). The location was defined as follows: upper and lower borders of the root-entry zone were determined by the uppermost and lowest fibers of the IX/X nerve bundle entering the medulla; the anterior border of the root-entry zone was defined as the transition of the olivary convexity to the concavity of the retro-olivary sulcus; and the posterolateral border was located at the junction of parenchymal brain tissue and individual nerve fibers. ${ }^{2}$ Arterial compression (vertebral artery or posteriorinferior cerebellar artery) within this defined area was considered 'positive' for RVLM, and patients with no arterial compression were considered 'negative.' We defined arterial compression as deformation of the medulla surface. Therefore, instances in which the artery just contacted the surface of the medulla were excluded from the criteria for RVLM vascular compression. The presence of RVLM vascular compression was evaluated by two neurologists who were unaware of each patient's medical history.

\section{Statistical analysis}

Data are shown as the mean \pm s.d. or median (minimum-maximum) for continuous variables. Statistical analysis for comparison of the two groups was performed using Student's $t$-test or the Mann-Whitney $U$-test for continuous variables and the $\chi^{2}$ test or Fisher's exact test for categorical variables. $P$-values $<0.05$ were considered statistically significant. Receiver operating characteristic curves were configured to establish cut-off points for SV that optimally predicted RVLM compression.

\section{RESULTS}

Of the 86 enrolled patients, 11 did not undergo MRI, 4 had missing data, 3 received thrombolysis, 4 had an NIHSS score $\geqslant 23$ on admission, 5 received anti-hypertensive or vasopressor medicines, a

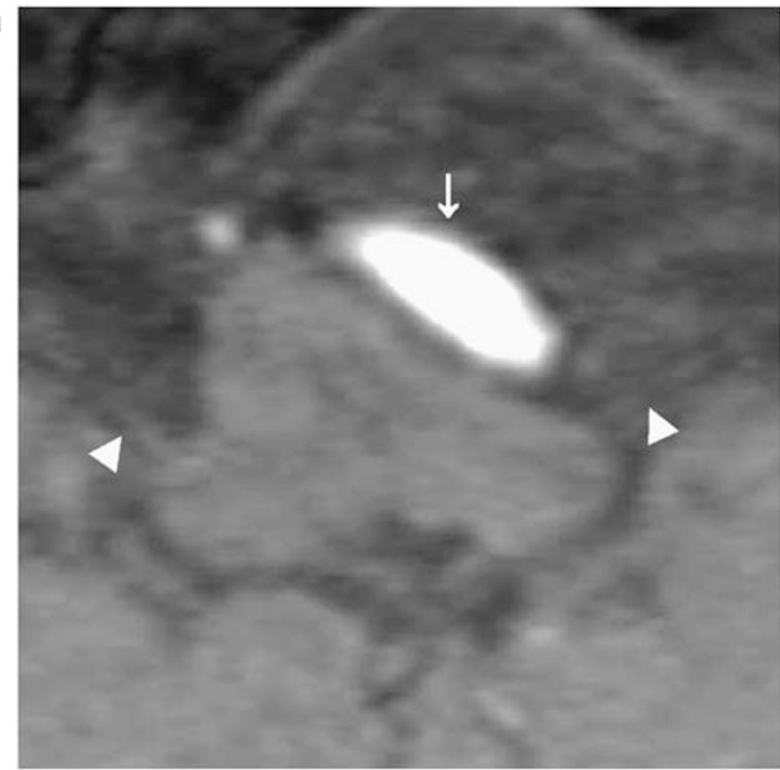

b

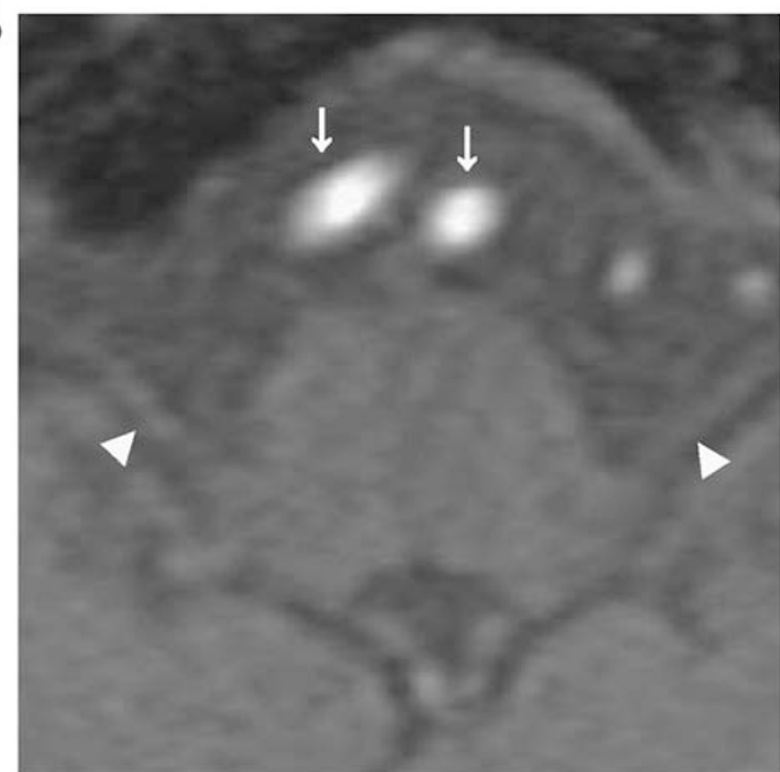

Figure 1 Axial 3D time-of-flight (TOF) images are shown (a) compression case, (b) non-compression case. The RVLM extends in a craniocaudal direction from the root entry zone of cranial nerve IX down to the upper part of cranial nerve XI. The RVLM is dorsolateral to the olive and medioventral to the root entry zone of cranial nerves IX and $X$ (arrowhead). The compression of vertebral artery (arrow) or posterior-inferior cerebellar artery within this defined area was considered 'positive' for RVLM.

and 3 had surgery within $48 \mathrm{~h}$ of hospital admission. Thus, all analyses were performed on the remaining 56 patients. MRI was performed at a mean time of $32.3 \mathrm{~h}$ after symptom onset (range, $4-50 \mathrm{~h}$ ). Table 1 shows the baseline characteristics of all patients. Vascular compression on the RVLM was identified in 15 (26.8\%) patients (hereafter, referred to as the compression group). Age was not significantly different between the patients with and without RVLM vascular compression. In the compression group, the proportion of males was significantly higher ( 93.3 vs. $63.4 \%, P=0.03$ ). The prevalence of hypertension was significantly higher in the compression group (93.3 vs. $61.0 \%$, $P=0.02$ ). At the time of admission, there was no significant difference 
Table 1 Baseline characteristics, blood pressure and NIHSS on admission, and ischemic stroke subtypes

\begin{tabular}{|c|c|c|c|}
\hline Variables & $\begin{array}{l}\text { Compression } \\
\quad(\mathrm{n}=15)\end{array}$ & $\begin{array}{l}\text { Non-compression } \\
\qquad(\mathrm{n}=41)\end{array}$ & P-value \\
\hline Age, mean years \pm s.d. & $71.6 \pm 9.37$ & $73.2 \pm 9.5$ & 0.59 \\
\hline Male, n (\%) & $14(93.3 \%)$ & $26(63.4 \%)$ & 0.03 \\
\hline \multicolumn{4}{|l|}{ Risk factors } \\
\hline Hypertension, n (\%) & $14(93.3 \%)$ & $25(61.0 \%)$ & 0.02 \\
\hline Diabetes mellitus, n (\%) & $9(60.0 \%)$ & $17(41.5 \%)$ & 0.22 \\
\hline Hyperlipidemia, n (\%) & $7(46.7 \%)$ & $27(65.9 \%)$ & 0.19 \\
\hline Baseline NIHSS & $4(0-8)$ & $4(0-20)$ & 0.29 \\
\hline Baseline SBP, mean $\mathrm{mm} \mathrm{Hg} \pm$ s.d. & $159.1 \pm 8.9$ & $149.5 \pm 26.1$ & 0.17 \\
\hline Baseline DBP, mean $\mathrm{mm} \mathrm{Hg} \pm$ s.d. & $81.1 \pm 14.7$ & $75.4 \pm 15.2$ & 0.88 \\
\hline \multicolumn{4}{|l|}{ Ischemic stroke subtype } \\
\hline Atherothrombotic, n (\%) & $5(33.3 \%)$ & $13(31.7 \%)$ & \\
\hline Cardioembolic, n (\%) & $2(13.3 \%)$ & $17(41.5 \%)$ & \\
\hline Small vessel, n (\%) & $3(20.0 \%)$ & $5(12.2 \%)$ & 0.17 \\
\hline Other etiology, n (\%) & $5(33.3 \%)$ & $6(14.6 \%)$ & \\
\hline
\end{tabular}

Abbreviations: DBP, diastolic blood pressure; NIHSS, National Institutes of Health Stroke Scale; SBP, systolic blood pressure.

Table 2 Relationship between RVLM vascular compression and clinical improvement at 14 days

\begin{tabular}{lccc}
\hline & $\begin{array}{c}\text { Improved (NIHSS } \\
4 \text { improvement or } \\
\text { NIHSS }=0)\end{array}$ & Unimproved & $\begin{array}{c}\text { OR }(95 \% \mathrm{Cl}) \\
\text { probability }\end{array}$ \\
\hline Compression & $4(26.7 \%)$ & $11(73.3 \%)$ & $0.21(0.06-0.78)$ \\
Non-Compression & $26(63.4 \%)$ & $15(36.6 \%)$ & $P=0.01$ \\
\hline
\end{tabular}

Abbreviations: $\mathrm{CI}$, confidence interval; NIHSS, National Institutes of Health Stroke Scale; RVLM, rostral ventrolateral medulla.

in NIHSS scores, and no significant difference was observed in systolic or diastolic blood pressure between the groups. In addition, the subtype of ischemic stroke was not significantly different between the groups $(P=0.17)$.

The patients were defined as clinically improved when their NIHSS score decreased more than four points or recovered to 0 on day 14 (ref. 19). The proportion of clinically improved patients was $26.7 \%$ in the compression group and $63.4 \%$ in the non-compression group (Table 2), with a significantly lower rate of improvement in the compression group (odds ratio, 0.21 (95\% $\mathrm{CI}=0.06-0.78) ; P=0.01$ ).

Figures 2 and 3 show the systolic and diastolic blood pressures and the SV values of the patients up to $72 \mathrm{~h}$ after admission. At all time points, no significant differences were observed in mean systolic or diastolic blood pressures between the two groups. However, the SV value of the systolic blood pressure was significantly higher in the compression group during the first $24 \mathrm{~h}$ after admission $(P<0.0001)$ and during the $72 \mathrm{~h}$ period after admission $(P<0.0001)$. Conversely, there were no significant differences in the diastolic blood pressure SV value between the groups at any time point. The pulse rate SV value was significantly higher in the compression group during the $72 \mathrm{~h}$ period after admission $(8.7 \pm 2.7$ b.p.m. vs. $7.3 \pm 2.2$ b.p.m., $P<0.05)$

In the explanatory analysis, the best cutoff $\mathrm{SV}$ value of the systolic blood pressure during the $72 \mathrm{~h}$ period after admission obtained from the Receiver operating characteristic curve was $15.3 \mathrm{~mm} \mathrm{Hg}$, which
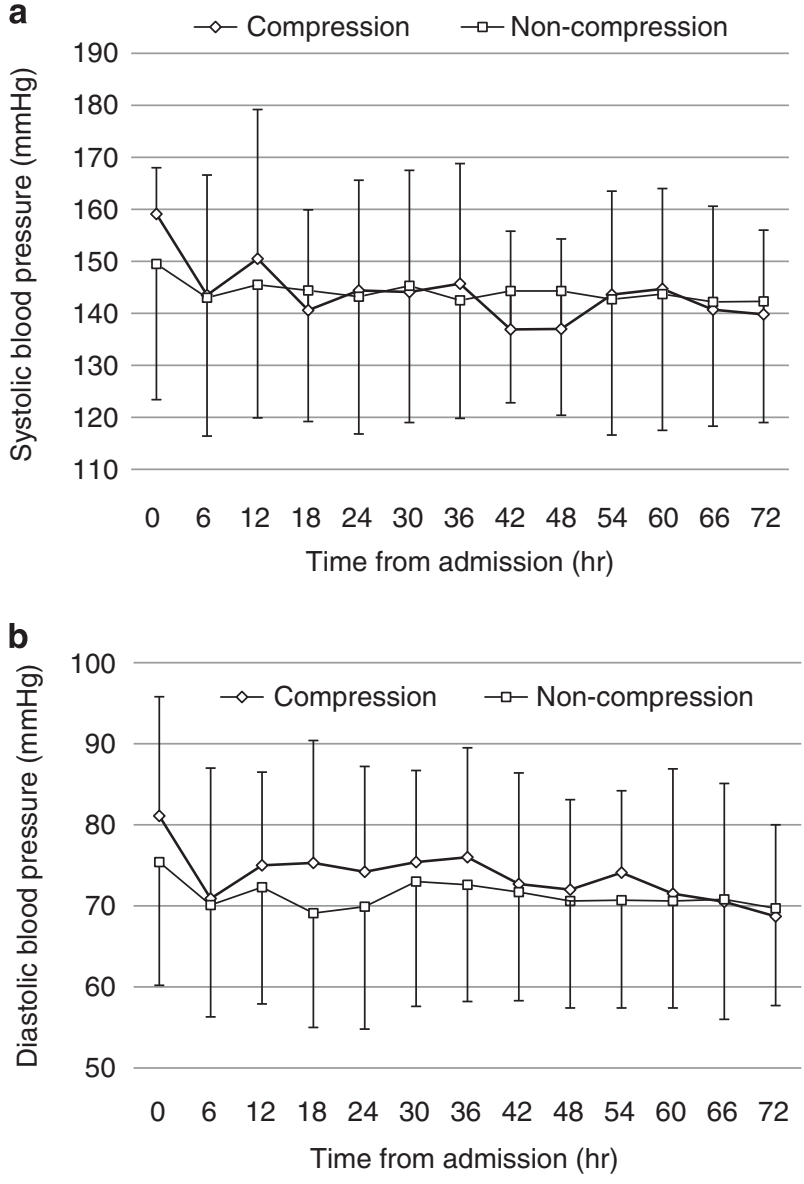

Figure 2 Average blood pressures during $72 \mathrm{~h}$ of hospitalization in the compression group $(n=15)$ and the non-compression group $(n=41)$. (a) Systolic blood pressure, (b) diastolic blood pressure.

predicted RVLM compression with a sensitivity of $67 \%$ and a specificity of $88 \%$ (area under the curve $=0.783 ; P=0.0002$ ). In the compression group, the patients with SV $<15.3 \mathrm{~mm} \mathrm{Hg}$ tended to show a more favorable outcome, defined using modified Rankin scale, than the patients with SV $\geqslant 15.3 \mathrm{~mm} \mathrm{Hg}(P=0.10)$ (Figure 4).

\section{DISCUSSION}

This study found that blood pressure variability during the acute ischemic stroke phase was significantly greater in patients with RVLM vascular compression than in those without. In addition, decreased improvement in neurologic symptoms was observed in patients with RVLM vascular compression compared with patients without RVLM vascular compression. In the compression group, patients with high $\mathrm{SV}$ values were more likely to have unfavorable outcomes. The association between blood pressure variability and acute ischemic stroke outcome has previously been reported. ${ }^{12,13}$ To our knowledge, this study is the first to clearly show an association between RVLM vascular compression, blood pressure variability and functional prognosis.

It has been reported that a large proportion of patients with RVLM vascular compression have hypertension. ${ }^{1-4}$ Mechanical stimulation of the RVLM causes glutamate release from RVLM vasomotor neurons, thereby increasing sympathetic nerve activity. ${ }^{20}$ RVLM vascular compression was observed in $7-22.2 \%$ of healthy individuals without 

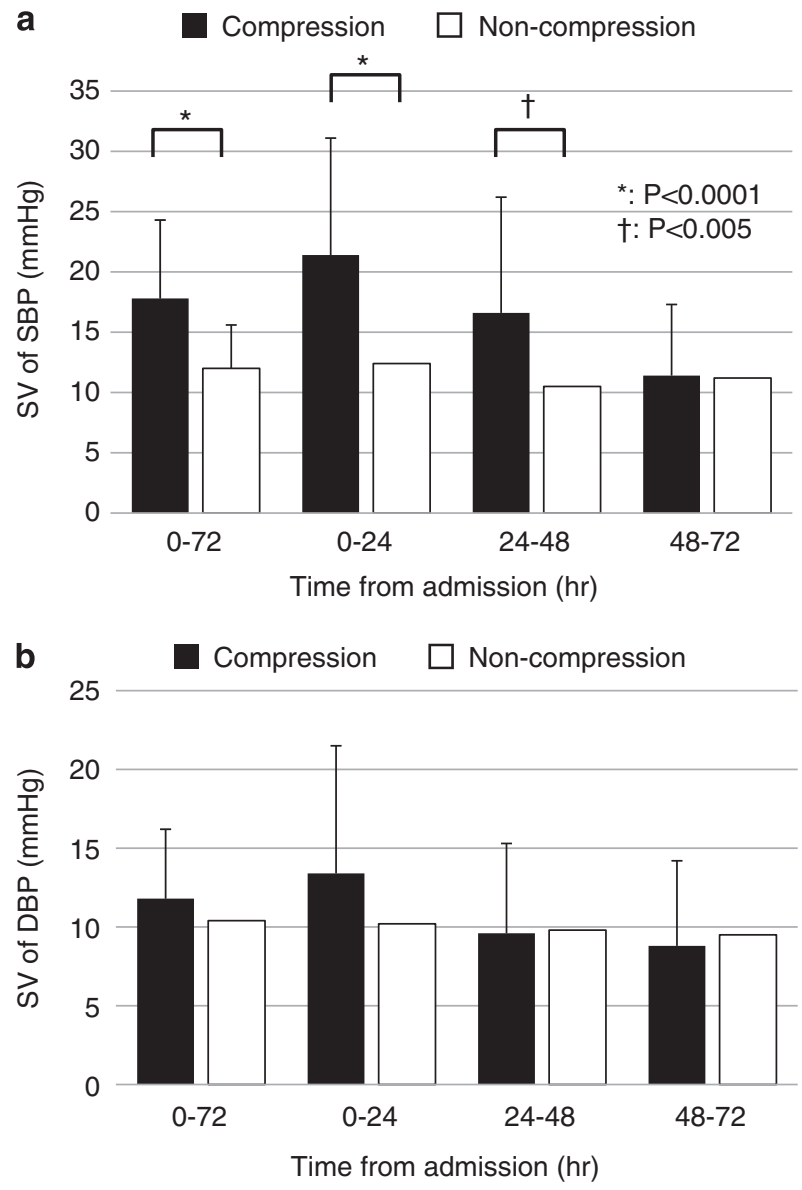

Figure 3 Successive variation (SV) of blood pressure during $72 \mathrm{~h}$ of hospitalization in the compression group $(n=15)$ and the non-compression group ( $n=41)$. (a) Systolic blood pressure (SBP), (b) diastolic blood pressure (DBP).

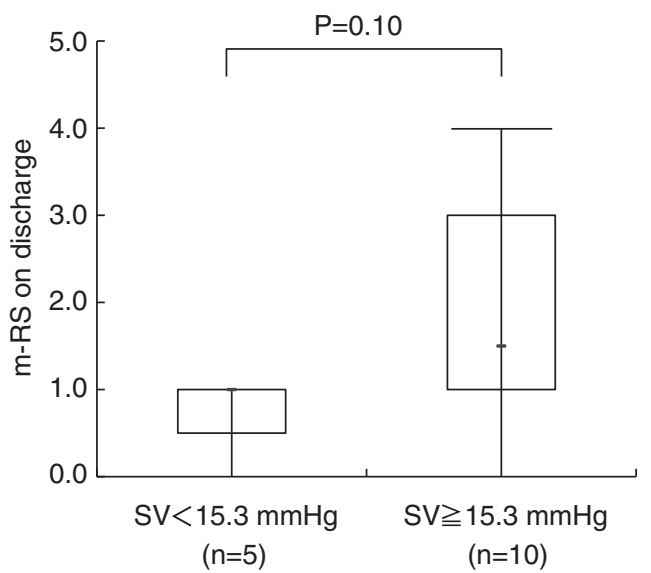

Compression group patients

Figure 4 Box plot showing median modified Rankin scale (m-RS) on discharge (solid bar), interquartile range (bar width), and minimum/maximum values across $\mathrm{SV}<15.3 \mathrm{~mm} \mathrm{Hg}$ or $\geqslant 15.3 \mathrm{~mm} \mathrm{Hg}$ in compression group.

hypertension and in $74-90 \%$ of patients with hypertension. ${ }^{3,4,21,22}$ Our results demonstrated that the prevalence of hypertension was 93.3\% (14/15) in patients with RVLM vascular compression, and it was higher than that in patients without RVLM vascular compression, which is consistent with previous studies. The prevalence of hypertension was $60.5 \%$ (25/41) in patients without RVLM vascular compression, which is similar to other previous population-based studies of ischemic stroke. ${ }^{23,24}$ We propose that RVLM vascular compression is one of the important factors related to hypertension. However, it is still unknown whether RVLM vascular compression is the cause of hypertension because no study has prospectively followed young normotensive subjects with RVLM vascular compression to evaluate the incidence of hypertension. Conversely, several reports have demonstrated that surgical decompression of the RVLM reduced sympathetic nerve activity and normalized systemic blood pressure. ${ }^{5,6}$ In addition, in our study, the patient with RVLM vascular compression who did not show hypertension $(n=1)$ had higher blood pressure variability than patients without RVLM vascular compression and had a poor prognosis on discharge. In the other target organs (the heart), it has been reported that the regression of left ventricular hypertrophy depends on not only blood pressure levels, but also the reduction of cardiac sympathetic drive. ${ }^{25}$ Therefore, we think that chronic sympathetic nerve activation influences target organ damage even when patients are not hypertensive.

In the present study, the compression group had a significantly higher SV value and a significantly lower proportion of clinically improved patients. In the compression group, the sympathetic nerve may be chronically activated. Hypertension is likely one of the markers of this chronic sympathetic nerve activation, which explains the significantly higher rate of hypertension in the compression group. In patients with chronic hypertension, cerebral blood flow decreases rapidly with cerebral ischemia in association with an increasing cerebral oxygen extraction fraction. ${ }^{26}$ Therefore, blood pressure variability may change cerebral blood flow. This pathological response may deteriorate the improvement of neurological symptoms in patients with RVLM vascular compression.

Our findings demonstrated that the difference in blood pressure between the groups was not significant, although it tended to be slightly higher in the compression group. Interestingly, the variability in blood pressure was significantly greater in the compression group. The sympathetic nervous system is generally persistently activated during acute ischemic stroke, leading to increased blood pressure regardless of the presence or absence of RVLM vascular compression. Therefore, it is difficult to detect a difference in blood pressure with or without RVLM vascular compression during the acute ischemic stroke phase. By contrast, RVLM vascular compression clearly activates the sympathetic nervous system, leading to increased burst-like firing of sympathetic nerves, ${ }^{27}$ which is thought to have a role in blood pressure variability.

It has been reported that patients with RVLM vascular compression have increased sympathetic nerve activity. ${ }^{8}$ In addition, the increased sympathetic nerve activity may induce high blood pressure variability because it was reduced after ganglion blockade with trimethaphan. ${ }^{28}$ In parallel to this response, when sympathetic nerve activity increases, the baroreflex counteracts this activation in normal subjects. However, it is still unknown whether the increase in sympathetic nerve activity is caused by increased sympathetic nerve traffic, increased vascular sensitivity, or impaired baroreflex buffering in patients with RVLM vascular compression. It has been reported that baroreflex buffering and baroreflex-mediated vasopressin release are severely impaired in patients with RVLM vascular compression. ${ }^{7}$ When the impaired baroreflex is associated with RVLM vascular compression, blood pressure variability may increase with increased sympathetic nerve activity. It has been suggested that restoration of baroreflex sensitivity may prevent stroke in the animal model. ${ }^{29}$ It is possible that the 
restoration of baroreflex sensitivity reduced blood pressure variability and, therefore, stroke incidence. However, blood pressure variability was not examined in the experiment by Liu et al. Therefore, it is unknown whether stroke prevention with the restoration of baroreflex sensitivity was mediated by the reduction of blood pressure variability. In our study, we evaluated the association of blood pressure variability at the acute ischemic stroke phase and its prognosis for patients with or without RVLM vascular compression. However, we were unable to evaluate blood pressure variability at the pre-ischemic stroke period. Therefore, further studies are needed to clarify the association between blood pressure variability and the occurrence of stroke in patients with RVLM vascular compression.

Various measures of variability of individual blood pressure profiles have been used. The most common measures of variations are the extreme values, such as maximum, minimum, range (difference between maximum and minimum), s.d., or coefficient of variation (s.d. over mean).$^{30}$ In the present study, we selected SV as a parameter of within-patient blood pressure variability. This parameter includes the serial variation on a time sequence, whereas other measures, such as s.d. and coefficient of variation, ignore the sequential nature of such a data set. Therefore, when analyzing the sequential nature of data, as in the present study, it is better to use SV. If not, time-invariant measures, such as s.d. or coefficient of variation, can result in a misleading prediction of prognosis and is less informative for blood pressure management.

In the present study, we evaluated RVLM vascular compression with MRI images. However, it is possible that cases in which there appeared to be RVLM vascular compression had a low degree of compression that did not result in sympathetic nerve activation. Thus, we examined the association between SV and prognosis in the compression group, which was divided into two groups using the best cutoff SV value of the systolic blood pressure during the $72 \mathrm{~h}$ period after admission. As a result, the patients with high SV values tended to show more unfavorable prognosis than the patients with low SV values. Therefore, large-scale studies are required to confirm the influence of the SV value on the compression group prognosis.

The present study has several limitations. First, a selection bias may exist because of the small sample size at a single institution. Therefore, it will be necessary to conduct a similar multi-center study to confirm whether these results can be generalized. In addition, our results may not reflect all ischemic stroke patients because of the frequent exclusion of severe cases. It is highly possible that the severe cases have poor prognoses regardless of the presence of RVLM vascular compression or that the influence of RVLM vascular compression might be low. To study these issues, we are planning a multi-center, large-scale trial to define the association of RVLM vascular compression with the prognosis of ischemic stroke patients. Another limitation of the current study is that we did not examine the correlation between RVLM vascular compression and SV value with the use of anti-hypertensive medications before stroke onset. In particular, medications that inhibit sympathetic nerve activation may influence blood pressure and blood pressure variability. However, the half-lives of these anti-hypertensive medications are not more than $24 \mathrm{~h}$, and their effects decrease with time. In our study, the SV value for blood pressure variability was calculated at $72 \mathrm{~h}$ after admission. Therefore, pre-medication with anti-hypertensives should have had a limited effect on the SV value. Finally, the correlation with ischemic location was not fully investigated. A previous study demonstrated that patients with infarctions involving the insular cortex tended to suffer from autonomic dysfunction, ${ }^{31}$ which may influence blood pressure. However, in the present study, few patients had infarctions involving the insular cortex, and the proportion of patients with infarctions involving the insular cortex was similar between the compression and non-compression groups (two in the compression group (13.3\%) and five in the non-compression group $(12.2 \%)$ ). Thus, the influence of ischemic location in the present study may also be limited.

In conclusion, this study found that patients with RVLM vascular compression had greater variability in blood pressure during the acute ischemic stroke phase, which may be a factor related to poorer prognosis. In the future, larger scale prospective studies are required to confirm the influence of RVLM vascular compression in acute ischemic stroke.

\section{ACKNOWLEDGEMENTS}

This study was supported in part by research grants from the Ministry of Health, Labor and Welfare, Japan.

1 Jannetta PJ, Segal R, Wolfson Jr SK. Neurogenic hypertension: etiology and surgical treatment. I. Observations in 53 patients. Ann Surg 1985; 201: 391-398.

2 Naraghi R, Gaab MR, Walter GF, Kleineberg B. Arterial hypertension and neurovascular compression at the ventrolateral medulla. A comparative microanatomical and pathological study. J Neurosurg 1992; 77: 103-112.

3 Naraghi R, Geiger H, Crnac J, Huk W, Fahlbusch R, Engels G, Luft FC. Posterior fossa neurovascular anomalies in essential hypertension. Lancet 1994; 344: 1466-1470.

4 Morimoto S, Sasaki S, Miki S, Kawa T, Itoh H, Nakata T, Takeda K, Nakagawa M, Kizu O, Furuya S, Naruse S, Maeda T. Neurovascular compression of the rostral ventrolateral medulla related to essential hypertension. Hypertension 1997; 30: 77-82.

5 Frank H, Schobel HP, Heusser K, Geiger H, Fahlbusch R, Naraghi R. Long-term results after microvascular decompression in essential hypertension. Stroke 2001; 32: 2950-2955.

6 Frank H, Heusser K, Geiger H, Fahlbusch R, Naraghi R, Schobel HP. Temporary reduction of blood pressure and sympathetic nerve activity in hypertensive patients after microvascular decompression. Stroke 2009; 40: 47-51.

7 Jordan J, Toka HR, Heusser K, Toka O, Shannon JR, Tank J, Diedrich A, Stabroth C, Stoffels M, Naraghi R, Oelkers W, Schuster H, Schobel HP, Haller H, Luft FC. Severely impaired baroreflex-buffering in patients with monogenic hypertension and neurovascular contact. Circulation 2000; 102: 2611-2618.

8 Sendeski MM, Consolim-Colombo FM, Leite CC, Rubira MC, Lessa P, Krieger EM. Increased sympathetic nerve activity correlates with neurovascular compression at the rostral ventrolateral medulla. Hypertension 2006; 47: 988-995.

9 Bath P, Chalmers J, Powers W, Beilin L, Davis S, Lenfant C, Mancia G, Neal B, Whitworth J, Zanchetti A. International society of hypertension (ISH): statement on the management of blood pressure in acute stroke. J Hypertens 2003; 21: 665-672.

10 Toyoda K, Okada Y, Fujimoto S, Hagiwara N, Nakachi K, Kitazono T, Ibayashi S, Iida M. Blood pressure changes during the initial week after different subtypes of ischemic stroke. Stroke 2006; 37: 2637-2639.

11 Shiokawa O, Sadoshima S, Fujii K, Yao H, Fujishima M. Impairment of cerebellar blood flow autoregulation during cerebral ischemia in spontaneously hypertensive rats. Stroke 1988; 19: 615-622.

12 Stead LG, Gilmore RM, Vedula KC, Weaver AL, Decker WW, Brown Jr RD. Impact of acute blood pressure variability on ischemic stroke outcome. Neurology 2006; 66: 1878-1881.

13 Yong $\mathrm{M}$, Kaste $\mathrm{M}$. Association of characteristics of blood pressure profiles and stroke outcomes in the ECASS-II trial. Stroke 2008; 39: 366-372.

14 Pagani M, Lombardi F, Guzzetti S, Rimoldi O, Furlan R, Pizzinelli P, Sandrone G, Malfatto G, Dell'Orto S, Piccaluga E, Turiel M, Baselli G, Cerutti S, Malliani A. Power spectral analysis of heart rate and arterial pressure variabilities as a marker of sympatho-vagal interaction in man and conscious dog. Circ Res 1986; 59: 178-193.

15 Adams Jr HP, Bendixen BH, Kappelle LJ, Biller J, Love BB, Gordon DL, Marsh III EE. Classification of subtype of acute ischemic stroke. Definitions for use in a multicenter clinical trial. TOAST. Trial of Org 10172 in Acute Stroke Treatment. Stroke 1993; 24: 35-41.

16 Brott T, Adams Jr HP, Olinger CP, Marler JR, Barsan WG, Biller J, Spilker J, Holleran R, Eberle R, Hertzberg V, Rorick M, Moomaw CJ, Walker M. Measurements of acute cerebral infarction: a clinical examination scale. Stroke 1989; 20: 864-870.

17 van Swieten JC, Koudstaal PJ, Visser MC, Schouten HJ, van Gijn J. Interobserver agreement for the assessment of handicap in stroke patients. Stroke 1988; 19: 604-607.

18 Schachinger $\mathrm{H}$, Langewitz W, Schmieder RE, Ruddel $\mathrm{H}$. Comparison of parameters for assessing blood pressure and heart rate variability from non-invasive twenty-four-hour blood pressure monitoring. J Hypertens 1989; 7 (suppl 3): S81-S84.

19 National Institute of Neurological Disorders, Stroke rt-PA Stroke Study Group. Tissue plasminogen activator for acute ischemic stroke. N Engl J Med 1995; 333. 1581-1587 
20 Morimoto S, Sasaki S, Miki S, Kawa T, Nakamura K, Ichida T, Itoh H, Nakata T, Takeda $\mathrm{K}$, Nakagawa M, Yamada H. Pressor response to compression of the ventrolateral medulla mediated by glutamate receptors. Hypertension 1999; 33: 1207-1213.

21 Akimura T, Furutani Y, Jimi Y, Saito K, Kashiwagi S, Kato S, Ito H. Essential hypertension and neurovascular compression at the ventrolateral medulla oblongata: MR evaluation. AJNR Am J Neuroradiol 1995; 16: 401-405.

22 Pierpaolo L, Mastronardi L, Strano S, Di Biasi C, Trasimeni G. Neurovascular conflict and essential arterial hypertension: MR evaluation. AJNR Am J Neuroradiol 1996; 17: 195-196.

23 Petty GW, Brown Jr RD, Whisnant JP, Sicks JD, O'Fallon WM, Wiebers DO. Ischemic stroke subtypes: a population-based study of incidence and risk factors. Stroke 1999; 30: 2513-2516.

24 Kolominsky-Rabas PL, Weber M, Gefeller O, Neundoerfer B, Heuschmann PU. Epidemiology of ischemic stroke subtypes according to TOAST criteria: incidence, recurrence, and long-term survival in ischemic stroke subtypes: a population-based study. Stroke 2001; 32: 2735-2740.

25 Morgan HE, Baker KM. Cardiac hypertrophy. Mechanical, neural, and endocrine dependence. Circulation 1991; 83: 13-25.
26 Temma T, Kuge Y, Sano K, Kamihashi J, Obokata N, Kawashima H, Magata Y, Saji H. PET 0-15 cerebral blood flow and metabolism after acute stroke in spontaneously hypertensive rats. Brain Res 2008; 1212: 18-24.

27 Schobel HP, Frank H, Naraghi R, Geiger H, Titz E, Heusser K. Hypertension in patients with neurovascular compression is associated with increased central sympathetic outflow. J Am Soc Nephrol 2002; 13: 35-41.

28 Zhang R, Iwasaki K, Zuckerman JH, Behbehani K, Crandall CG, Levine BD. Mechanism of blood pressure and R-R variability: insights from ganglion blockade in humans. J Physiol 2002; 543: 337-348.

29 Liu AJ, Ma XJ, Shen FM, Liu JG, Chen H, Su DF. Arterial baroreflex: a novel target for preventing stroke in rat hypertension. Stroke 2007; 38: 1916-1923.

30 Rothwell PM, Howard SC, Dolan E, O'Brien E, Dobson JE, Dahlöf B, Sever PS, Poulter NR. Prognostic significance of visit-to-visit variability, maximum systolic blood pressure, and episodic hypertension. Lancet 2010; 375: 895-905.

31 Tokgozoglu SL, Batur MK, Top uoglu MA, Saribas O, Kes S, Oto A. Effects of stroke localization on cardiac autonomic balance and sudden death. Stroke 1999; 30: 1307-1311. 